DOI:10.2478/jbcr-202O-OO18

Original Article

\title{
PREVENTION AND TREATMENT OF POTENTIALLY PROBLEMATIC ACUTE WOUNDS TO ACHIEVE FULL RECOVERY BY USING PLATELET- RICH PLASMA: RESEARCH RESULTS
}

\author{
Tsvetan V. Sokolov \\ Clinic of Orthopaedics and \\ Traumatology, \\ Kanev University Hospital - Ruse
}

\section{Corresponding Author:}

Tsvetan Sokolov

Clinic of Orthopedics and Traumatology,

Kanev University Hospital - Ruse

2, Nezavisimost Str.

Ruse, 7002

Bulgaria

e-mail: sokolovi2oo1@abv.bg

Received: September 25, 2020

Revision received: October 21, 2020

Accepted: December 11, 2020

\section{Summary}

The study presents the outcomes from platelet-rich plasma (PRP) application in treating acute and potentially problematic skin wounds from 2009 to 2016. Fifty-six hospitalized patients with acute skin wounds were divided into two groups: an experimental group of 30 patients treated with platelet-rich plasma and 26 controls with similar wounds, treated by conventional methods. After platelet-rich plasma treatment of 30 acute and potentially problematic wounds in the Experimental Group patients, 29 (96.7\%) of the wounds healed for 16 weeks on average. After applying conventional treatment with surgical wound debridement, of the 26 acute, potentially problematic wounds in the controls, only $2(7.6 \%)$ healed. Since acute and potentially problematic wounds are very likely to become chronic and difficult to heal, we recommend PRP application as a preventive treatment in patients at risk.

Keywords: platelet-rich plasma, acute and potentially problematic skin wounds, chronic wounds

\section{Introduction}

The skin is the largest organ in the human body. It plays a significant role in sustaining life by maintaining the water-electrolyte balance, regulating temperature, and acting as a barrier against various harmful external agents, including microorganisms. When this barrier is broken due to wounds, burns, neoplasms, or trauma, these functions can no longer be sustained adequately. This is why it is so important to restore the integrity of the skin as soon as possible. Skin ulcers represent tissue loss from the epidermis and derma, and sometimes they affect adipose tissue and muscle fascia. If spontaneous recovery is not achieved, a tissue lesion turns into a fibrous scar [1,2]. It has been proven that $15 \%$ of acute skin wounds become chronic [3].

Problematic skin wounds (PSW) cover a broad range of injuries, including acute, potentially problematic, such as in cases of high energy trauma and elderly patients. A wide variety of intrinsic and extrinsic factors interfere with healing, and PSWs are hard to heal and turn into chronic skin wounds (CSW). Acute skin wounds (ASW) affect skin integrity and sometimes the underlying tissues, and the recovery process follows strictly defined consecutive biology stages. ASWs are particularly difficult to treat because 
they are located in bone prominences, underlying tendons, plantar surface of the foot, and around joints. When there are skin defects, the latter can turn into potentially problematic skin wounds [1]. The wound healing process involves a strictly defined set of consecutive events, which starts at the time of injury and lasts for several months. It could be divided into three phases: inflammation, proliferation, and remodeling (differentiation) $[4,5,6,7]$.

The patient's venous blood produces autologous platelet-rich plasma (PRP), which consists of growth factors (GF), cytokines, fibrin scaffold, and chemokines. Molecular and cellular induction of normal wound healing is achieved by adding GF from PRP to the wound, similar to that observed when platelets are activated [1].

The purpose of our research was to evaluate the outcomes of platelet-rich plasma application in treating acute skin wounds.

\section{Materials and Methods}

The research took place from 2009 to 2016 . There were 56 hospitalized patients with acute skin wounds in total. The experimental group included 30 patients treated with platelet-rich plasma. The control group consisted of 26 patients with similar wounds, treated by conventional methods such as surgical wound debridement. A detailed interview was conducted with patients with clarifying questions on the therapy applied. Informed consent for treatment with PRP was obtained from the 30 patients included in the experimental group. The remainder were put in the control group. According to the Helsinki Declaration's principles, all surveyed participants in the study signed their informed consent [8].

Wounds were assessed based on scores introduced by Cancela AM [1]. Total Wound Score (TWS), total anatomic score (TAS) of a wound, and Total Score of Wound data (TSWD) were used to evaluate specific wound parameters.

The data obtained were analyzed using the software SPSS (Statistical Package for the Social Sciences). Non-parametric tests (Kolmogorov-Smirnov, Mann-Whitney, and Wilcoxonwere) were also used to verify the significance of relations between features. The level of significance for $p$ was assumed to be at least 0.05 [9].

All patients in the experimental group received treatment with PRP derived from PRGF Endorfet, based on a treatment algorithm we designed. The first step was surgical treatment and thorough wound debridement. On the second postoperative day, we applied PRP to the wound by infiltrating wound edges and adding PRP to the wound bed. PRP was prepared as follows: blood was drawn in blood collection tubes with sodium citrate. The tubes were then centrifuged for 8 minutes at $1800 \mathrm{r} / \mathrm{min}$. Plasma was drawn with micropipettes, and a $10 \% \mathrm{CaCl}_{2}$ activator was added. The ratio was $1 \mathrm{ml}$ plasma to $50 \mu \mathrm{l}$ activator. After 5 minutes, the PRP obtained became active. In about 40 minutes, a fibrin clot was formed. Wound edges were infiltrated with the activated plasma in a liquid state, while the fibrin clot was used to fill the wound. Then the wound was dressed with a soft bandage soaked in saline. This procedure was repeated every seven days until the wound had entirely healed.

Table 1 shows systematic data about the

Table 1. Distribution of EG patients based on anatomic location of ASW, infectious agent and wound depth

\begin{tabular}{lclclc}
\hline \multicolumn{2}{c}{ DEPTH } & \multicolumn{2}{c}{ LOCATION } & \multicolumn{2}{c}{ AGENT } \\
\hline Anatomic location & Number & Anatomic location & Number & Anatomic location & Number \\
\hline hypodermis & 11 & Achilles & 2 & S. aureus & 9 \\
\hline fascia & 9 & knee & 5 & E. coli & 5 \\
\hline tendon & 3 & lateral malleolus & 6 & P.aeruginosa & 1 \\
\hline bone & 7 & ankle & 6 & K. pneumoniae & 1 \\
\hline & shin & 7 & E. faecalis & 1 \\
\hline & heel & 2 & & \\
\hline & shoulder & 1 & & \\
\hline & palm & 1 & & \\
\hline
\end{tabular}


Table 2. Distribution of EG patients based on anatomic location of ASW, infectious agent and wound depth

\begin{tabular}{|c|c|c|c|c|c|}
\hline \multicolumn{2}{|c|}{ DEPTH } & \multicolumn{2}{|c|}{ LOCATION } & \multicolumn{2}{|c|}{ AGENT } \\
\hline Anatomic location & Number & Anatomic location & Number & Anatomic location & Number \\
\hline hypodermis & 10 & Achilles & 2 & S. aureus & 9 \\
\hline fascia & 8 & knee & 4 & E. coli & 6 \\
\hline tendon & 3 & lateral malleolus & 5 & P. aeruginosa & 2 \\
\hline \multirow[t]{5}{*}{ bone } & 5 & ankle & 5 & K. pneumoniae & 2 \\
\hline & & shin & 6 & E. faecalis & 2 \\
\hline & & heel & 2 & & \\
\hline & & shoulder & 1 & & \\
\hline & & palm & 1 & & \\
\hline
\end{tabular}

experimental group (EG) patients with acute skin wounds based on anatomic location, infectious agent, and wound depth of ASW. The average area of wounds was $1490 \mathrm{~mm}^{2}$, and the patients' median age was 54.2 years. Ten patients had controlled type 2 diabetes.

Table 2 shows the control group (CG) patients' systematic data with acute skin wounds based on anatomic location, infectious agent, and wound depth. The average wound area was $1310 \mathrm{~mm}^{2}$, and the median age was 55.2 years. There were 8 patients with controlled type 2 diabetes in the $\mathrm{CG}$.

\section{Results}

After PRP treatment of 30 acute skin wounds of patients from the experimental group, all 29 wounds $(96.7 \%)$ healed for 16 weeks on average. No data proving wound recovery was found for one wound $(3.33 \%)$ in the experimental group.

After conventional treatment with surgical wound debridement of the 26 acute skin wounds in the control group, full recovery occurred only in two patients $(7.7 \%)$. No recovery was achieved in the remainder of $24(92.3 \%)$ patients.

Figure 1 shows the clinical case of a 40 -yearold man with traumatic arthrotomy on the left ankle after an accident with a chainsaw. The patient underwent surgical treatment of the wound and tendoraphy of extensor tendons. On the 14th day after the trauma, a wound with a $400 \mathrm{~mm}^{2}$ area formed, with $10 \mathrm{~mm}$ wound edges. PRP treatment protocol was initiated. The photos show the recovery process dynamics and full recovery on week 16. Figure 2 shows the ASW treatment trend of the same patient by using different wound assessment scores. The microbiological wound tests isolated S. aureus. No antibiotics were administered.

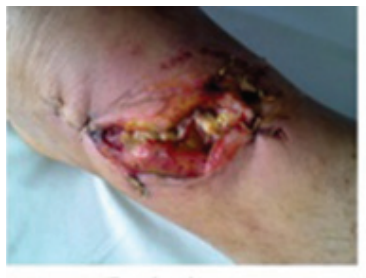

Beginning

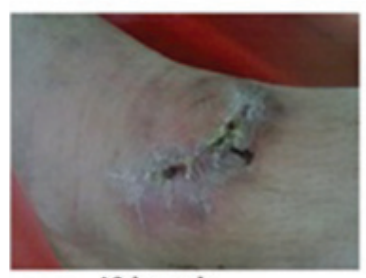

12th week

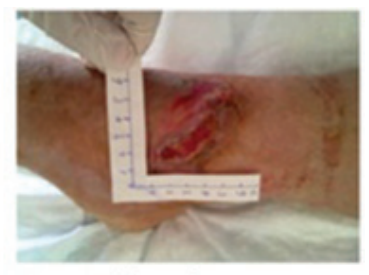

4th week

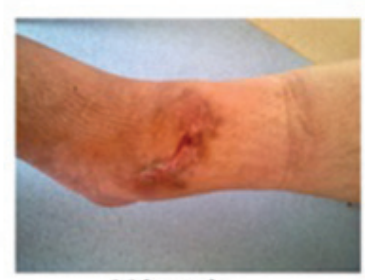

16th week
Figure 1. Clinical course of ASW healing with PRP treatment of a 40-year-old man, successfully treated for 16 weeks

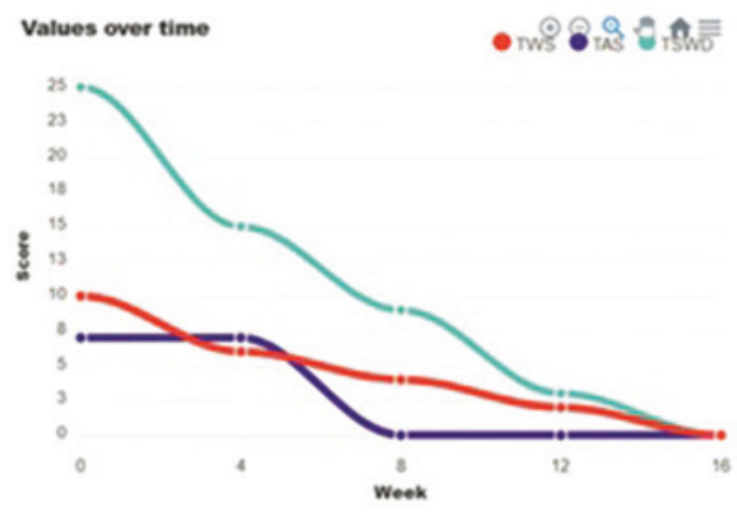

Figure 2. Treatment trend of acute skin wounds of the same patient 
Figure 3 shows the clinical case of a 17 -yearold woman with wound dehiscence after surgery for hemangioma in the Achilles area. On the 12 th day after surgery, a wound with an area of $300 \mathrm{~mm}^{2}$ formed, with $5 \mathrm{~mm}$ wound edges. PRP treatment protocol was started. The photos illustrate the dynamics of the recovery process. Full recovery was seen on week 12 . Figure 4 shows the ASW treatment trend of the same patient by using different wound assessment scores. The microbiological wound tests isolated S. Aureus. No antibiotics were administered.

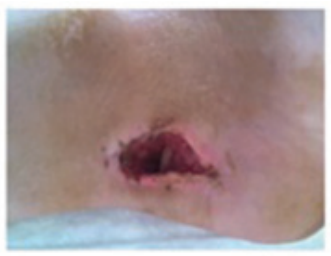

Beginning

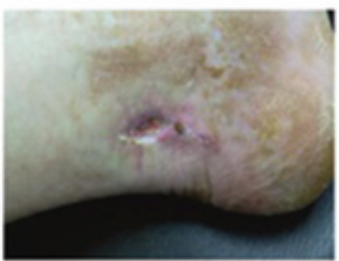

8 th week

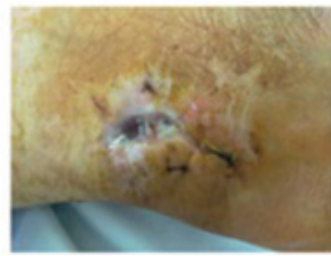

4th week

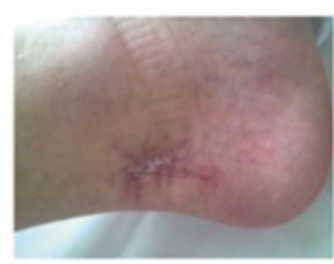

12th week
Figure 3. Clinical course of ASW healing with PRP treatment of a 17-year-old woman, successfully treated for 16 weeks

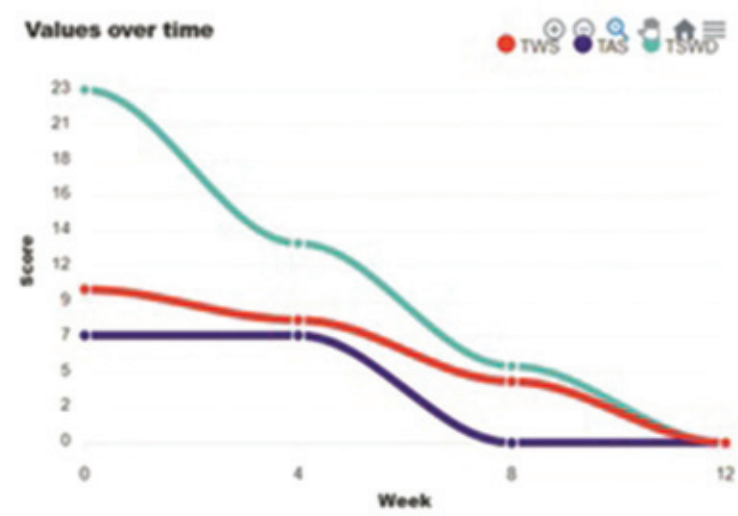

Figure 4. Treatment trend of acute skin wounds of the same patient

Figure 5 shows a 62-year-old woman's clinical case with a bimalleolar fracture, metal osteosynthesis, and a skin defect. On the second day after the surgery, a wound of 560 $\mathrm{mm}^{2}$ formed with wound edges of $3 \mathrm{~mm}$. PRP treatment protocol was initiated. The photos

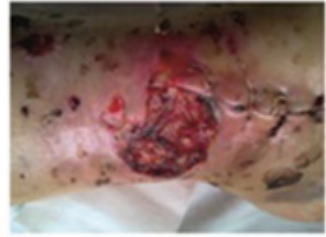

Beginning

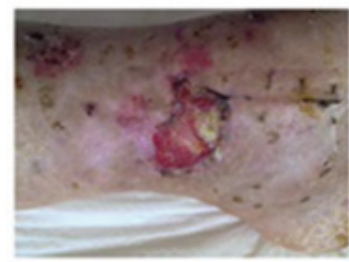

4th week

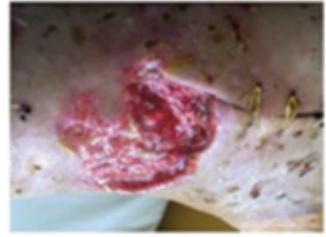

wound debridement

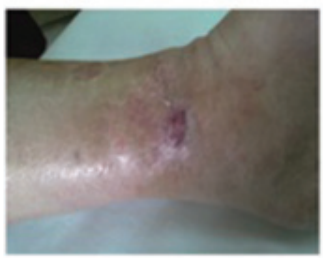

8th week
Figure 5. Clinical course of ASW healing with PRP treatment of a 62-year-old woman, successfully treated for 16 weeks

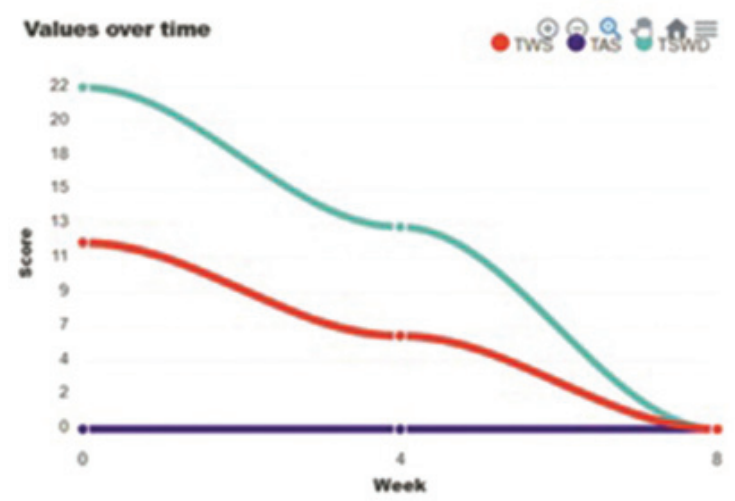

Figure 6. Treatment trend of acute skin wounds of the same patient

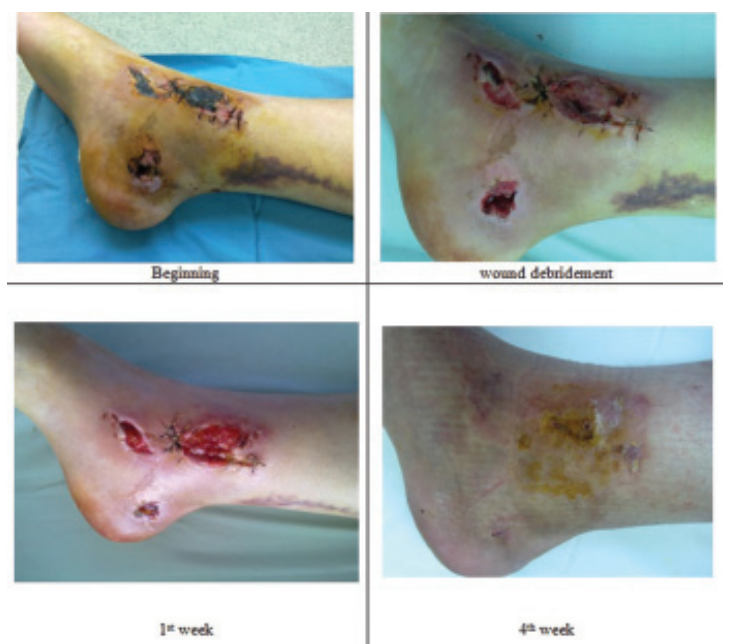

Figure 7. Clinical course of ASW healing with PRP treatment of a 40-year-old man, successfully treated for 4 weeks

show the recovery process dynamics and full recovery on week 8 . Figure 6 shows the ASW treatment trend of the same patient by using 
wound assessment scores. The microbiological wound tests isolated S. Aureus. No antibiotics were administered.

Figure 7 shows a 42-year-old man's clinical case with a bimalleolar fracture, metal osteosynthesis, and a skin defect. On the second day after the surgery, a wound of $600 \mathrm{~mm}^{2}$ had formed with $4 \mathrm{~mm}$ wound edges. The PRP treatment protocol was applied. The photos illustrate the dynamics of the recovery process. Full recovery was achieved on week 4 .

\section{Discussion}

The results obtained and analyzed in our study showed that PRP application in acute, potentially problematic skin wounds PSW helps achieve excellent results. Treatment resulted in accelerating healing, shorter hospital stay, and minimizing wound complications. The prophylactic use of PRP on high-risk patients could contribute to full tissue recovery and prevent wounds from turning into chronic wounds that are difficult to heal. We aimed to determine whether the PRP method can be effectively used for acute and potentially problematic skin wounds. The PRP application results were compared with results in the control group, in which surgical wound debridement and a soft bandage dressing were applied. Comparing the other possible alternative methods for the treatment of such wounds will be made in another study.

Many sources prove PRP's positive effect and the benefits of its applications on the healing process of hard-to-heal wounds. Data on the application of PRP for the prevention and treatment of ASW is limited. Meta-analyses of studies on the treatment of ASW with PRP found the following:

Picard et al. report the results from applying PRP to support wound healing. The purpose of this study was to test whether using PRP would support the full recovery of acute wounds, burns, and laser therapy outcomes. Three randomized controlled acute wound studies have statistically demonstrated benefits on healing time, time to return to work, quality of life, and pain. Three prospective controlled studies have found a statistically significant difference in terms of healing time. Authors have concluded that PRP application enhanced ASW healing process [10].
Wang et al. evaluated the PRP efficacy for the treatment of acute wounds. Many studies have been reviewed, including 13 reports on 982 patients. Meta-analyses results have shown that healing time was shorter in the PRP group than the control group with no PRP. PRP may reduce ASW healing time and hospitalization time. It may also minimize wound healing complications and the need for blood transfusion and may relief posttraumatic pain. Furthermore, PRP has a positive effect on wound infection control [11].

Another PRP application in the PSW treatment process is to prevent and treat wound infection. Numerous sources have proved the positive effect of PRP treatment of wound infections.

El-Sharkawy et al. analyzed GF in PRP and the effects of PRP application on the release of cytokines from monocytes and lipoxin A4. The authors concluded that PRP is a rich source of growth factors and supports significant changes in the monocyte-mediated proinflammatory release of cytokines and chemokines. PRP may inhibit cytokine release, limit inflammation, and promote tissue regeneration because of increased lipoxin A4 [12].

Cieslik-Bielecka et al. reported that PRP in the form of gel inhibits the growth of S. aureus and E.coli, but no similar effect on wounds with K1.pneumoniae, E.faecalis, and Ps.aeruginosa. No correlation between leukocytes and platelet concentrations and antimicrobial activity was observed. The activity of antimicrobial peptides of platelets against fungi was less pronounced as compared to the activity against bacteria. Gel PRP induced in vitro growth of Ps. Aeruginosa which means that PRP may exacerbate infections with this agent. Authors consider that the combination of gel-like PRP inductive and antimicrobial properties may enhance recovery of infected delayed adhesion or non-adhesion [13].

In their study, Anitua et al. have reported on the antimicrobial potential of the growth factorrich plasma when used against methicillinsensitive and methicillin-resistant S.aureus and S.epidermidis. They also report the effect of incorporated leucocytes in growth factor-rich plasma. Blood samples were obtained from five healthy volunteers and used to prepare each type of plasma rich in growth factors (F1, F3, F3+leu). The Microbiological activity of growth factorrich plasma against two staphylococcal strains 
was tested, based on counting surviving bacterial colonies after incubation for 0.4 and 8 hours with various forms. The authors found out that all types had a bacteriostatic effect, especially in the first 4 hours of administration. All types had an antibacterial effect on the $4^{\text {th }}$ hour on three out of four strains, except for methicillin-sensitive S.epidermidis. The researchers concluded that plasma rich in growth factors could be used to fight and prevent postoperative wound infections [14].

Our study also supports these results from PRP application for PSW treatment, reviewing which risk patients are suitable for the treatment method [15].

The use of the respective antimicrobial, antibiotic treatment for an acute and potentially problematic skin wound depends on the derived microbiological research. The laboratory results determine the use of only one or more antibiotics. The duration of antibiotic use depends on the antimicrobial agent and the respective antibiotic, to which the former is susceptible.

Patients from EG and CG fall into age groups in which change in biological status is due to physiological changes. One of the factors contributing to the development of PSW is the patients' advanced age. The skin of elderly patients is easily injured. There is also a lack of adequate stress response regarding gene regulation of stress-related proteins $[16,17,18]$. There are numerous changes in the cellular and molecular characteristics of mature skin, which inhibit the wound healing process $[19,20]$. The normal wound healing process in elderly patients is disrupted by delayed cell proliferation, changes in production and composition of the extracellular matrix, and different responses to growth factors. They, alone or in combination, may contribute to a rise in incidence and duration of wounds in elderly patients [16].

The PRP, however, is not a panacea. The modern treatment of acute skin wounds and especially contaminated wounds does not include only wounds debridement but also other therapeutic methods. We switch to alternative therapies such as various skin flaps, different medications, electrical stimulation, mechanical stimulation-vacuum aspiration, cell-based therapy (epithelial stem cells), gene therapy, or hyperbaric oxygenation, in the event of a poor outcome of PSW treatment with PRP [21,22,23].

This research was conducted on a limited number of patients with acute skin wounds, treated within the research period at the Clinic of Orthopedics and Traumatology, after signing informed consent. Patients with problematic skin wounds might be treated with PRP in other surgical healthcare facilities, which is to be part of future research.

\section{Conclusions}

The use of activated platelets from PRP for treating PSW is a preventive and therapeutic method that dramatically supports the tissuebuilding process. The physical recovery and normal wound healing process are enhanced by delivering necessary growth factors, which play a major role. ASW often turn into hard-toheal and chronic wounds, and we recommend preventive use of PRP in patients at risk.

\section{Acknowledgments}

The article reflects the results of the work on project No. 2020-FOZZG-02 "Development of algorithms for diagnosis and treatment of problematic skin wounds by the method of enriched platelet-rich plasma", funded by the Research Fund, Ruse University.

\section{References}

1. Cancela AM, Lana JF, Annichino-Bizzachi JM. Use of Platelet-Rich Plasma (PRP) in Treating Chronic Wounds. In: Lana JF et al.(eds). PlateletRich Plasma. Lecture Notes in Bioengineering. 2014;281-8.

2. Crovetti G, Martinelli G, Issi M, Barone M, Guizzardi M, Campanati B. Platelet gel for healing cutaneous chronic wounds. Transfus Apher Sci. 2004;30(2):145-51.

3. Lindholm C, Searle R. Wound management for the 21 st century: combining effectiveness and efficiency. Int Wound J. 2016;Suppl 2:5-15.

4. Carter MJ, Fylling CP, Parnell LK. Use of Platelet Rich Plasma Gel on Wound Healing: A Systematic Review and Meta-Analysis. Eplasty. 2011;11:e38.

5. Clark RAF. Overview and general considerations of wound repair. In: Clark RAF (eds). The molecular and cellular biology of wound repair. Plenum Press. New York. 1996;3-50. 
6. Marx RE. Platelet-rich plasma: a source of multiple autologous growth factors for bone grafts. In: Lynch SE et al. (eds.), Tissue engineering, applications in maxillofacial surgery and peiodontics. 1999;71-82.

7. Perez AGM, Lana JF, Rodrigues AA, Luzo ACM.Challenges and a Feasible Strategy for Studies and Standardization of Platelet-Rich Plasma In: Lana JF, et al. (eds.), Platelet-Rich Plasma. Lecture Notes in Bioengineering. 2014;119-36.

8. World Medical Association. World Medical Association Declaration of Helsinki. Ethical principles for medical research involving human subjects. Bull World Health Organ. 2001;79:373-4.

9. Lazarov B, Karakoleva S. Using GAS syntax for education in Mathematics. In: Higher education in Bulgaria and the Europe 2020 strategy. 2011;3:914-21.

10. Picard F, Hersant B, Bosc R, Meningaud JP.Should we use platelet-rich plasma as an adjunct therapy to treat ,acute wounds, “" „burns, “" and „laser therapies“: A review and a proposal of a quality criteria checklist for further studies. Wound Repair Regen. 2015;23(2):163-70.

11. Wang $\mathrm{L}, \mathrm{Gu} \mathrm{Z}$, Gao C. Platelet rich plasma for treating acute wound: a meta-analysis. Zhonghua Yi Xue Za Zhi. 2014;94(28):2169-74.

12. El-Sharkawy H, Kantarci A, Deady J, Hasturk H, Liu H, Alshahat M. et al. Platelet-rich plasma: growth factors and pro- and anti-inflammatory properties. J Periodontol. 2007;78(4):661-9.

13. Cieslik-Bielecka A, Gazdzik TS, Bielecki TM, Cieslik T.Why the platelet-rich gel has antimicrobial activity? Oral Surg Oral Med Oral Pathol Oral Radiol Endod. 2007;103(3):303-5.

14. Anitua E, Alonso R, Girbau C, Aguirre JJ, Muruzabal F, Orive G. Antibacterial effect of plasma rich in growth factors (PRGFEndoret) against Staphylococcus aureus and Staphylococcus epidermidis strains. Clin Exp Dermatol. 2012;37(6):652-7.
15. Sokolov Ts., Manukova A., Stefka Karakoleva S., Valentinov B.,Petrova N. Analysis of the results of applying the method platelet-rich plasma (PRP) for the treatment of problematic skin wounds. J of IMAB. 2017;23(1):1460-65.

16. Martin P, Nunan R. Cellular and molecular mechanisms of repair in acute and chronic wound healing. British Journal of Dermatology. 2015;173:370-8.

17. Mustoe T. Understanding chronic wounds: A unifying hypothesis on their pathogenesis and implications for therapy. Am J Surg. 2004;187(5A):65-70.

18. Nunan R, Harding K, Martin P. Clinical challenges of chronic wounds: searching for an optimal animal model to recapitulate their complexity. Dis Model Mech. 2014;7(11):120513.

19. Enoch S, Harding K. Wound Bed Preparation: The Science Behind the Removal of Barriers to Healing Wounds. 2003;15(7):213-29.

20. Harries RL, Bosanquet DC, Harding KG. Wound bed preparation: TIME for an update. Int Wound J. 2016;Suppl 3:8-14.

21. Matkov O, Kovachev V, Vasilev V, Kovachev M. Microvascular surgery in orthopedic and traumatology, plastic and reconstructive surgery - results and prospects. Angiology and Vascular Surgery. 2015;2:48-50.

22. Lim CS, Baruah M, Bahia SS. Diagnosis and Management of Venous Leg Ulcers. BMJ. 2018;362:k3115.

23. Mizzi A, Cassar K, Bowen C, Formosa C. The progression rate of peripheral arterial disease in patients with intermittent claudication: a systematic review. J Foot Ankle Res. 2019;12(1):40. 